\title{
Why are left-right spatial codes easier to form than above-below ones?
}

\author{
ROBERTO NICOLETTI \\ Università di Padova, Padova, Italy \\ CARLO UMILTÀ \\ Università di Parma, Parma, Itally \\ and \\ EMANUELE PATRIZIO TRESSOLDI and CARLO ALBERTO MARZI \\ Università di Padova, Padova, Italy
}

\begin{abstract}
Previous studies have shown that spatial compatibility is stronger in the left-right than in the above-below dimension. This left-right dominance cannot be attributed to a better representation of the effectors in left-right than in above-below locational codes or to the fact that incompatible left-right stimulus-response pairings cross the body midline, whereas incompatible above-below ones do not. Nicoletti and Umiltà (1985) proposed that the left-right dominance should be attributed to the allocation of attention to the more difficult discrimination, which, in vision, is that concerning the left-right dimension. This attentional hypothesis was tested in the present study, in which we used the auditory modality. We reasoned that because in the auditory modality the above-below discriminations are more difficult than left-right ones, attention should be preferentially allocated to the former. Therefore, in audition an above-below dominance should replace the left-right dominance observed in vision. Experiments 1 and 2 showed a clear-cut compatibility effect in the auditory modality for both the left-right and above-below dimensions. Experiment 3 showed that spatial compatibility was still stronger for the left-right than for the above-below dimension. Since the left-right one proved to be the more discriminable dimension, this finding rules out the attentional hypothesis, at least in the version originally proposed.
\end{abstract}

Among the varieties of stimulus-response compatibility (Nicoletti \& Umiltà, 1984; Simon, Sly, \& Vilapakkam, 1981), spatial compatibility is the one that yields the strongest effects. In it the selection of the correct response depends exclusively on the position of the stimulus. For example, the right-side stimulus requires a rightside response (compatible pairing) or a left-side response (incompatible pairing). Of course, the compatibility effect is shown by the fact that reaction time (RT) is faster for the compatible than for the incompatible pairings. The most convincing explanation of spatial compatibility is in terms of a match (compatible pairings) or a mismatch (incompatible pairings) between the binary spatial codes that specify the relative positions of stimuli and responses (Nicoletti, Anzola, Luppino, Rizzolatti, \& Umiltà, 1982; Nicoletti \& Umiltà, 1984; Nicoletti, Umiltà, \& Ladavas, 1984; Wallace, 1971, 1972). In other words, it is assumed that upon presentation of the test stimulus its spatial code is formed and compared to that of the response. RT is faster when the two codes are the same than when they are different (see also Teichner \& Krebs, 1974).

Address correspondence to Roberto Nicoletti, Dipartimento di Psicologia Generale, Università di Padova, Piazza Capitaniato 3, 35139 Padova, Italy.
The spatial dimension most frequently employed in spatial compatibility studies is the left-right one, but recent work has shown that the same effects can be found by using the above-below dimension (see, e.g., Ladavas \& Moscovitch, 1984; Nicoletti \& Umiltà, 1984, 1985). This means that, when demanded by the task, above-below locational codes of both stimuli and responses can be obtained and matched, as happens for left-right codes. It is also worth noting that, if studied in isolation, abovebelow compatibility effects are of more or less the same magnitude as the left-right ones. Therefore, there seems to be no intrinsic difficulty in forming above-below spatial codes and using them for mapping stimuli into responses. Surprisingly, however, the situation changes radically when left-right cues also are present (Nicoletti \& Umiltà, 1984, 1985). When both left-right and abovebelow cues are available, the use of the latter apparently becomes more difficult or altogether impossible, as attested by the weakening (Nicoletti \& Umiltà, 1984) or the disappearance (Nicoletti \& Umiltà, 1985) of the compatibility effects in the above-below dimension.

One obvious explanation of the dominance of left-right spatial cues is that, when the subject uses the hands for responding, the effectors are more easily described in terms of left-right than above-below positions. However, Nicoletti and Umiltà (1985) were able to disprove this ex- 
planation by replicating the left-right dominance effect in one experiment in which the responses were emitted with one hand and one foot instead of the two hands. It would seem that the most natural way of describing the relative positions of hand and foot is through abovebelow, rather than left-right, spatial representations.

A second likely explanation comes to mind if one considers that left-right locations are usually defined across the body midline, whereas the above-below distinction does not refer to any fixed reference axis. It may be that it is the availability of such an unambiguous reference axis in the left-right dimension that renders the left-right spatial cues comparatively more salient than the above-below cues. This explanation also can be rejected, however, because it is known (Nicoletti et al., 1982; Umiltà \& Nicoletti, 1985) that spatial compatibility effects are of the same magnitude irrespective of whether the left-right cues are defined across the body midline.

Recently, Nicoletti and Umiltà (1985) proposed an attentional interpretation of the left-right dominance. The rationale of the hypothesis is as follows. Since, for a horizontally symmetrical organism, discriminating between left and right is rather difficult (see, e.g., Corballis \& Beale, 1983), it is possible that attention is allocated to the left-right dimension, thus rendering left-right spatial cues more salient. This interpretation is similar to that proposed for the so-called visual dominance (see, e.g., Posner, Nissen, \& Klein, 1976): Human beings are thought to allocate attention to the visual modality, at the expense of other modalities, because visual stimuli are more difficult to detect.

Another example in which it seems that attention is preferentially allocated to the more difficult of two tasks can be found in an experiment on the interference between vocal and manual responses to the same stimulus (Holender, 1980). Subjects were presented with one of four possible letters and the task was to emit either a single response (vocal or manual) or a double response (vocal and manual). It was found that in the single-response condition RT was slower for the manual than for the vocal response, whereas in the double-response condition the opposite was true. This result is interesting here because it can be interpreted in terms of differential allocation of attention. One can argue that the manual response mode was more difficult than the vocal one (hence the slower RT in the single-response condition), but when the two responses had to be emitted simultaneously, attention was preferentially allocated to the more difficult response mode, and, as a result, RT became faster for manual than for vocal responses.

If the attentional hypothesis is correct, we should expect a stronger spatial compatibility effect for the abovebelow than for the left-right dimension when the abovebelow spatial cues become more difficult to discriminate than the left-right ones. There is evidence (Broadley \& Kirkland, 1979; Gardner \& Gardner, 1979; Oldfield \&
Parker, 1984) that sound localization is more difficult in the vertical than in the horizontal plane. The reason for this is not entirely clear. The most accepted explanation is that, due to the anatomical structure of the ears and their relative location, the source of a sound is localized more easily when the task requires discriminating between left and right than between above and below positions. It has also been noted, however, that human beings have specific dedicated mechanisms at low levels of the nervous system (e.g., the superior olive and other brainstem structures) that can perform rapid left-right auditory discrimination by comparing input from the two ears. No analogous low level mechanisms exist for vertical auditory discrimination. Therefore, above-below position discrimination involves a much higher level analysis of sensory input, which is largely monaural if the subject is not permitted head movements. This no doubt can cause slower abovebelow than left-right discrimination. At any rate, in experimental conditions identical to those of the previous studies (Nicoletti \& Umiltà, 1984, 1985), but in acoustic rather than visual stimuli are employed, one would expect subjects to allocate attention preferentially to the above-below dimension, which, in the auditory modality, is the more difficult discrimination. As a result, the abovebelow spatial cues should become comparatively more salient and spatial compatibility effects should become stronger in the above-below than in the left-right dimension.

In accordance with this line of reasoning, the rationale of the series of experiments reported here was the following. Experiments 1 and 2 were aimed at demonstrating left-right and above-below spatial compatibility effects, respectively, in isolation. In Experiment 3, left-right and above-below cues were combined orthogonally in order to assess the relative magnitude of the two types of spatial compatibility when both sets of cues were available. It was reasoned that the attentional hypothesis would gain support if the results showed that spatial compatibility was now stronger in the above-below than in the left-right dimension. In other words, we expected a reversal of the effect found in our previous studies (Nicoletti \& Umiltà, 1984, 1985).

\section{EXPERIMENT 1}

Experiments 1 and 2 were not strictly necessary, because it was already known that compatibility effects can be found in the auditory modality for both the left-right (Simon, 1969) and above-below (Simon, Mewaldt, Acosta, \& Hu, 1976) dimensions. However, considering that there have been very few such studies, and not all of them have dealt with spatial compatibility proper (see Nicoletti \& Umiltà, 1984; Simon et al., 1981, for a distinction among the various types of compatibility), we wanted to make sure that those findings could be replicated in our experimental conditions. Furthermore, for 
the purpose of the present study it was important to get a baseline measure of left-right and above-below compatibility effects in isolation. Experiment 1 measured left-right compatibility effects in the auditory modality.

\section{Method}

Subjects. The subjects were 8 students at the Università di Padova, who ranged in age from 20 to 23 years. All were righthanded and were naive as to the purpose of the experiment.

Apparatus. The experiment took place in a sound-attenuated cubicle. Each subject sat in front of a panel containing two identical loudspeakers fastened to a movable bar, one $50 \mathrm{~cm}$ to the right and the other $50 \mathrm{~cm}$ to the left (about $29^{\circ}$ ) of the subject's body midline. The distance between the head of the subject and the panel was $100 \mathrm{~cm}$, as was the distance between the loudspeakers. The subject positioned his/her head in an adjustable head-and-chinrest, and held with each hand a brass cylinder with a pushbutton on top. The two cylinders were placed along a horizontal bar and the distance between them was $50 \mathrm{~cm}$. The acoustic stimuli (duration of $500 \mathrm{msec}$, intensity of $70 \mathrm{~dB}$, and frequency of $1000 \mathrm{~Hz}$ ) were generated by a Commodore CBM 832 computer and delivered through the two loudspeakers. The interstimulus interval was $10 \mathrm{sec}$. The response latencies were recorded to the nearest millisecond by an electronic counter that was started with the onset of the stimulus and stopped by the switch press.

Procedure. The subjects were tested in three sessions, one for practice and two for data collection. In the practice session, half were compatible and half incompatible trials. The two data collection sessions (one for compatible and the other for incompatible trials) were subdivided into two blocks, separated by a 3-min rest period. During each block (40 trials), the acoustic stimulus was delivered by either the left- or the right-side loudspeaker, according to a random sequence. The subject was instructed to press one of the two pushbuttons with his/her thumb as fast as possible following the onset of the stimulus; the instructions stressed both speed and accuracy, but, except for the practice trials, no feedback was given. After the first block, both the loudspeakers and the buttons were switched to ensure careful matching. In the compatible condition the subject was required to respond to the left-side sound with the left-side button and to the right-side sound with the rightside button, whereas in the incompatible condition the instructions were reversed so that the left-side sound was paired with the rightside button and the right-side sound was paired with the left-side button. The order of conditions was counterbalanced across the subjects. Errors were discarded but not replaced.

Before presenting the results, we should point out that sound localization depends on several factors, that is, interaural phase differences, intensity differences, and differences in the sound shadow produced by the pinnae. Admittedly, it is not clear what mediated sound localization in the present and in the following experiments. However, the important point is that sound localization could not depend on spurious factors such as differences in sound quality between the two loudspeakers.

\section{Results and Discussion}

The correct RTs were submitted to a two-way repeated measures analysis of variance (ANOVA) in which the factors were stimulus position (left and right) and response position (left and right). As expected, the interaction reached significance $[F(1,7)=112.78, p<.001]$. The right-side button was pressed $74 \mathrm{msec}$ faster than the left in response to the stimulus coming from the right ( 318 vs. $392 \mathrm{msec}$ ), whereas the left-side button was pressed $86 \mathrm{msec}$ faster than the right for the stimulus coming from the left ( 300 vs. $386 \mathrm{msec}$ ). Therefore, compatible responses were $80 \mathrm{msec}$ faster overall than incompatible ones ( 309 vs. $389 \mathrm{msec}$ ). Errors were about $6 \%$ and were not submitted to statistical analysis.

Overall, the results showed a clear-cut spatial compatibility effect, which replicated the findings of previous studies and also provided a baseline for the subsequent experiments.

\section{EXPERIMENT 2}

Experiment 2 replicated Experiment 1, with appropriate changes in stimulus and response locations: The two stimuli and the two responses were placed one above the other and aligned with the vertical midline of the body.

\section{Method}

Subjects. Eight new subjects participated in the experiment. They were selected as in Experiment 1 and their ages were between 19 and 28 years.

Apparatus. The apparatus was identical to that of Experiment 1, except for stimulus and response positions. The two loudspeakers were positioned one above the other, $50 \mathrm{~cm}$ (about $29^{\circ}$ ) above and below the subject's eyes, and the two response buttons were fastened to the opposite ends of a vertical bar $50 \mathrm{~cm}$ in height. Half of the subjects held the top cylinder with the right hand and the bottom one with the left hand, whereas for the other half the arrangement was reversed.

Procedure. The procedure exactly replicated that of Experiment 1 . In the compatible condition the instructions were to respond to the top sound with the top button and to the bottom sound with the bottom button, whereas in the incompatible condition the pairing was reversed.

\section{Results and Discussion}

Mean correct RTs were entered into a two-way repeated measures ANOVA in which the two factors were stimulus position (above and below) and response position (above and below).

The analysis showed a significant effect of stimulus position $[F(1,7)=6.40, p<.05]$, due to the fact that RT to the bottom sound was $32 \mathrm{msec}$ faster than that to the top sound ( 467 vs. $499 \mathrm{msec}$ ). Six subjects showed the effect, and for 1 there was no difference. Also, in this experiment, stimulus position interacted significantly with response position $[F(1,7)=16.73, p<.005]$. Responses with the top button were $59 \mathrm{msec}$ faster than those with the bottom button in responding to a sound coming from above ( 443 vs. $502 \mathrm{msec}$ ), whereas responses with the bottom button were $123 \mathrm{msec}$ faster than those with the top button in responding to a sound coming from below (432 vs. $555 \mathrm{msec}$ ). Therefore, compatible responses were $90 \mathrm{msec}$ faster overall than incompatible ones (438 vs. $528 \mathrm{msec}$ ). Errors were $8.9 \%$ and were not submitted to statistical analysis.

The main effect of stimulus position does not have a clear explanation. When the visual modality was employed, Nicoletti and Umiltà $(1984,1985)$ attributed the advantage for the bottom stimulus to the greater sensitivity of the upper hemiretinae, but now it is apparent that 
a more general explanation, not linked to the modality of presentation, must be sought. Of greater importance is the significant interaction, which shows that the spatial compatibility effect can also occur in the vertical dimension. This finding fulfilled the main condition for proceeding with Experiment 3. In addition, to compare Experiments 1 and 2, we ran a further ANOVA with experiment as a between-subjects factor and type of pairing (compatible and incompatible) as a within-subjects factor. The two main effects were significant $[F(1,14)=$ $11.24, p<.005$, and $F(1,14)=53.36, p<.001$, respectively]. The latter main effect simply showed that compatible pairings produce faster responses than incompatible pairings ( 373 vs. $459 \mathrm{msec}$ ). More interesting was the outcome of the comparison between experiments, which showed that overall, RT was $134 \mathrm{msec}$ slower in Experiment 2 than in Experiment 1 (483 vs. $349 \mathrm{msec}$ ). This finding is in agreement with previous work, which demonstrated that the localization of sound in the vertical plane is more difficult than in the horizontal plane (Broadley \& Kirkland, 1979; Gardner \& Gardner, 1973; Oldfield \& Parker, 1984).

\section{EXPERIMENT 3}

In this experiment the conditions were such that the left-right and above-below cues varied independently and orthogonally. In other words, the stimulus-response pairings could be either compatible or incompatible for both dimensions or compatible for one and incompatible for the other. This allowed an independent estimate of both types of compatibility in the presence of a competing pair of spatial cues, and not in isolation as in Experiments 1 and 2 .

\section{Method}

Subjects. Eight new subjects from the Università di Padova participated in the experiment. They ranged in age from 21 to 25 years and were selected as before.

Apparatus. The apparatus was as already described, but the positions of stimuli and response buttons differed due to a left-right displacement. This displacement was obtained by rotating to an inclination of $45^{\circ}$ the bar previously mentioned. The loudspeakers were thus positioned $35 \mathrm{~cm}$ to the right or left of the body midline, and $35 \mathrm{~cm}$ (about $20^{\circ}$ ) above or below the eyes. The response buttons were positioned $17.5 \mathrm{~cm}$ to the right or left of the body midline and $17.5 \mathrm{~cm}$ apart along the vertical dimension.

Procedure. The experimenter gave the instructions by pointing to the loudspeaker and the pushbutton, without labeling the stimulus or response positions. After one practice session, the subjects attended on four conditions, each divided into two contiguous blocks of 20 trials each, separated by a 1 -min rest period. In one condition (Condition $\mathbf{N}$ ), stimulus-response pairings were compatible for neither dimension. For instance, the bottom right sound required a response with the top left button, and the top left sound required a response with the bottom right button. Another condition (Condition V) comprised pairings that were compatible only for the vertical dimension (above-below). For example, the bottom right sound required a response with the bottom left button, and the top left sound required a response with the top right button. A third condition (Condition $\mathrm{H}$ ) contained pairings compatible only for the horizontal dimension (right-left). In this case, the bottom right sound

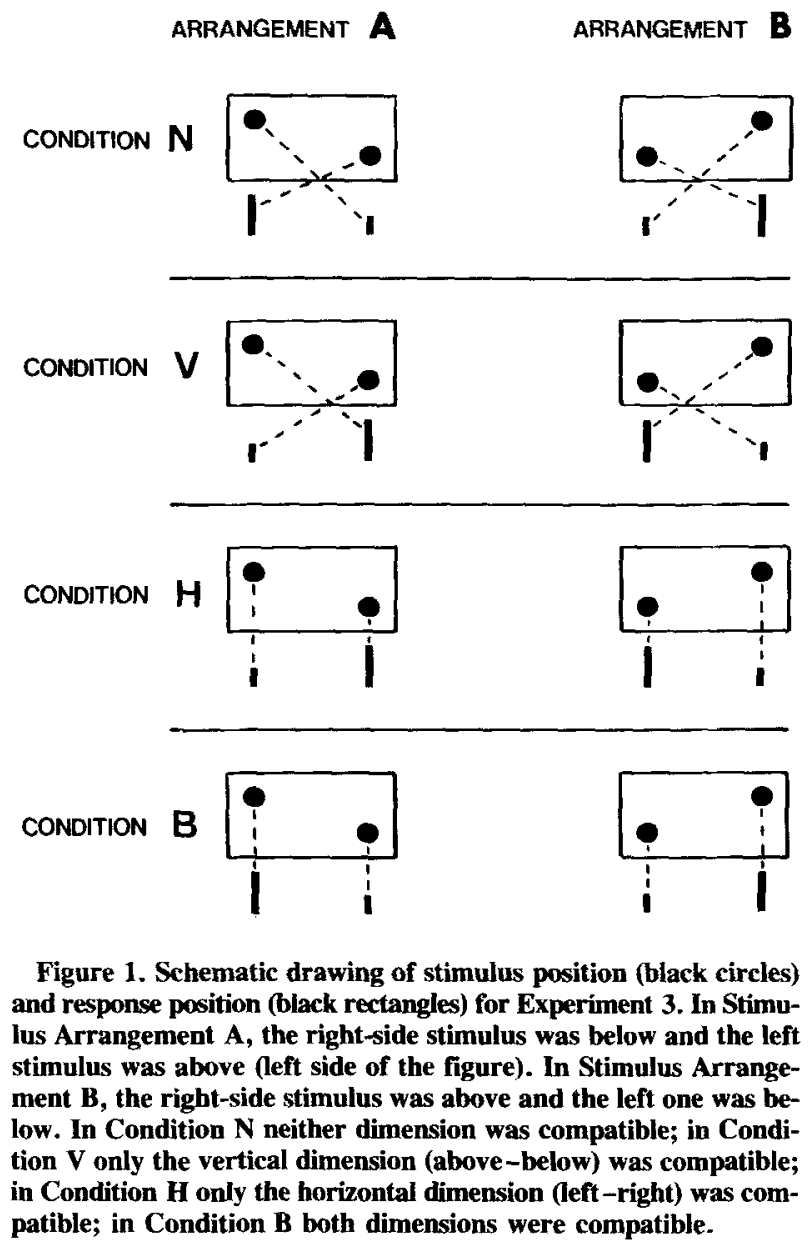

required a response with the top right button, and the top left sound required a response with the bottom left button. The fourth condition (Condition B) was compatible for both the above-below and left-right dimensions. For instance, the bottom right sound required a response with the bottom right button, and the top left stimulus required a response with the top left button (see Figure 1).

The examples above make reference to one of the two possible arrangements of the stimuli (i.e., Arrangement A in Figure 1). As shown in the figure, the two arrangements differed in that in one (Arrangement $\mathrm{A}$ ), one stimulus was in the bottom-right position and the other in the top-left position, whereas in the second arrangement (Arrangement B), the two stimulus positions were always topright and bottom-left. All subjects participated in the four conditions, but for half of them the arrangement was always of the type A, whereas for the others it was always of the type B. The order of presentation of the four conditions was counterbalanced across the subjects.

\section{Results and Discussion}

The simplest and clearest way to analyze the results is by considering the four experimental conditions (see Table 1). The RT data were submitted to a two-way ANOVA with one between-subjects factor, stimulus arrangement (type $A$ and type $B$ ), and one within-subjects factor, condition (Conditions $\mathrm{N}, \mathrm{V}, \mathrm{H}$, and $\mathrm{B}$ ). The main effect of condition was significant $[F(3,18)=18.5$, $p<.001]$. A post hoc set of comparisons with the 
Table 1

Mean Latencies (in msec), Standard Deviations (SD), and Percentage

Error Rates (PE) as a Function of the Main Experimental Conditions

\begin{tabular}{|c|c|c|c|c|c|c|c|c|c|c|c|}
\hline \multicolumn{3}{|c|}{ B } & \multicolumn{3}{|c|}{$\mathbf{H}$} & \multicolumn{3}{|c|}{ V } & \multicolumn{3}{|c|}{$\mathbf{N}$} \\
\hline$M$ & $S D$ & PE & $M$ & $S D$ & PE & $M$ & $S D$ & PE & $M$ & $S D$ & PE \\
\hline \multicolumn{12}{|c|}{ Experiment 1} \\
\hline- & - & - & 309 & 83.6 & 2.65 & - & - & - & 389 & 69.0 & 3.43 \\
\hline \multicolumn{12}{|c|}{ Experiment 2} \\
\hline - & - & - & - & - & - & 438 & 77.53 & 3.98 & 528 & 100.5 & 4.91 \\
\hline \multicolumn{12}{|c|}{ Experiment 3} \\
\hline 294 & 41.9 & 2.26 & 282 & 51.6 & 1.01 & 362 & 70.1 & 2.41 & 374 & 71.3 & 1.40 \\
\hline
\end{tabular}

Note-B = compatible for both dimensions, $\mathbf{H}=$ compatible for the horizontal dimension only,

$\mathrm{V}=$ compatible for the vertical dimension only, $\mathrm{N}=$ compatible for neither dimension.

Newman-Keuls method showed that RT was slower in Condition $\mathrm{N}$ than in Conditions $\mathrm{H}$ and $\mathrm{B}(p<.01 ; 374$ vs. 282 and $294 \mathrm{msec}$, respectively). Also, RT was slower in Condition $\mathrm{V}$ than in Conditions $\mathrm{H}$ and $\mathrm{B}(p<.01$; 362 vs. 282 and $294 \mathrm{msec}$, respectively). In essence, RT increased by $80 \mathrm{msec}$ when the left-right dimension was changed from compatible to incompatible (288 vs. $368 \mathrm{msec}$ ), whereas the same change in the above-below dimension had not influence on RT (328 $\mathrm{msec}$ in both cases). All subjects showed a much larger left-right than above-below compatibility effect (actually, the abovebelow compatibility effect was reversed in 5 subjects). Errors occurred in $7 \%$ of the trials and were not analyzed.

There can be little doubt that in the present experiment there was a strong spatial compatibility effect in the left-right dimension, whereas the effect was altogether absent in the above-below dimension. Furthermore, it is apparent that the presence of above-below cues had no influence whatsoever on left-right compatibility effect: the effect was $80 \mathrm{msec}$ irrespective of whether the cues were present or absent (see Table 1, Experiments 1 and 3 , respectively). In contrast, the left-right cues had a dramatic influence on the above-below compatibility effect, which was $90 \mathrm{msec}$ when they were absent (Experiment 2) and dropped to $0 \mathrm{msec}$ in their presence (Experiment 3).

In conclusion, the results of this experiment confirmed in full those of two very similar experiments in the visual modality (Nicoletti \& Umiltà, 1984, Experiment 4; 1985, Experiment 3). It is also clear that this outcome is in direct contradiction to the predictions of the attentional hypothesis we had proposed. In the auditory modality, the left-right dominance, instead of being replaced by the expected above-below dominance, became, if anything, even stronger.

\section{CONCLUSIONS}

The results of the present experiments, along with those of two previous similar studies (Nicoletti \& Umiltà, 1984, 1985), unambiguously lead to the following conclusions: (1) When presented with only left-right or only abovebelow positional cues, the subject is able to make use equally easily of either pair for mapping stimuli onto responses; and (2) the use of left-right cues eliminates any effect of the above-below cues.

It seems we are now in a position to reject all three possible explanations of the left-right dominance. It cannot be attributed to the fact that left-right positions are defined by the crossing of the body midline, because their encoding does not become less effective when the body midline is not crossed (Nicoletti et al., 1982; Umiltà \& Nicoletti, 1985). It cannot be attributed to the fact that the two hands are easier to encode in left-right terms, because the effect is still present when the responses are emitted with one hand and one foot (Nicoletti \& Umiltà, 1985). Finally, as the present study has shown, it cannot be attributed to the fact that the left-right discrimination is more difficult, and hence more attention demanding, because the effect is present even in the auditory modality, where the above-below discrimination is more difficult.

The left-right dominance effect becomes even more puzzling if one considers that left-right positions are usually much more difficult to discriminate than abovebelow positions (Corballis \& Beale, 1983), and that, in other experimental conditions, above-below positions are automatically encoded even though encoding is not required by the task (Simon et al., 1976).

Other instances of left-right dominance are not easy to find in the literature. Sholl and Egeth (1981, Experiment 3) found that, when no verbal mediation was involved, locational discriminations in the horizontal dimension were faster than those in the vertical dimension. However, this finding was not significant. To the best of our knowledge, there is only one clear instance of left-right dominance, that is, the left-right dominance in the perception of symmetry. It is well established (see, e.g., Corballis \& Beale, 1983, for a well-documented review) that symmetry in the left-right dimension is much more salient than symmetry in the above-below dimension. Furthermore, it seems that the salience of the left-right symmetry is only slightly diminished when the axis of symmetry is not aligned with the body midline.

Symmetry can be thought to play a role in the left-right dominance effect observed here, if one considers that in 
a spatial compatibility task the subject must form an internal representation of the spatial arrangement of stimuli and responses in order to be able to retrieve, under time pressure, the correct stimulus-response pairing. One can also speculate that the building up of the internal representation is guided by the global characteristics of the display, among which symmetry is one of the most prominent (Richards, 1978; Royer, 1981). Since left-right symmetry is more important than above-below symmetry, left-right cues become in turn more salient than abovebelow cues, hence the left-right dominance effect.

Another point worth considering is that in the case of the left-right discrimination, a very prominent reference axis is available, that is, the vertical body midline. The positions of both stimuli and responses are determined with reference to it. Conversely, in the case of the abovebelow discrimination, no such clear reference axis is available for defining the positions of stimuli and responses. It can be suggested that the availability of an unambiguous way of spatial encoding renders easier the mapping of stimuli onto responses, hence the left-right dominance effect. Of course, this is again the body midline hypothesis, which we refuted because it had been shown (Nicoletti et al., 1982) that the left-right compatibility effect was of the same magnitude regardless of whether the stimulus response pairings crossed the body midline. We reasoned that this hypothesis predicted that the left-right cues should have been more salient, and thus the spatial compatibility stronger, when the stimuli were presented and the responses emitted on opposite sides of the body midline. However, this finding becomes less damaging for the body midline hypothesis if one considers the possibility that the left-right axis continues to be effective even when it is not aligned with the body midline. That is to say, it is possible that any left-right discrimination is based on an egocentric axis, which can be either the body midline itself or a projection of the body midline into the external space.

\section{REFERENCES}

Broadley, G., \& KirkLAND, J. (1979). Sound localization accuracy on vertical and horizontal planes. Perceptual \& Motor Skills, 49, 354.

Corballis, M. C., Beale, I. L. (1983). The ambivalent mind. Chicago: Nelson-Hall.

Gardner, M. B., \& Gardner, R. S. (1973). Problem of localization in the median plane: Effect of pinnae cavity occlusion. Journal of the Acoustical Society of America, 53, 400-408.

HolENDER, D. (1980). Interference between a vocal and a manual response to the same stimulus. In G. E. Stelmach \& J. Requin (Eds.), Tutorials in motor behavior. Amsterdam: North-Holland.

Ladavas, E., \& Moscovitch, M. (1984). Must egocentric and environmental frames of reference be aligned to produce spatial S-R compatibility effects? Journal of Experimental Psychology: Human Perception \& Performance, 10, 205-215.

Nicoletti, R., Anzola, G. P., Luppino, G., Rizzolatti, G., \& UMILTÁ, C. (1982). Spatial compatibility effects on the same side of the body midline. Journal of Experimental Psychology: Human Perception \& Performance, 8, 664-673.

Nicoletti, R., \& UMıLtÀ, C. (1984). Right-left prevalence in spatial compatibility. Perception \& Psychophysics, 35, 333-343.

NicoleTtI, R., \& UMILTÁ, C. (1985). Responding with hand and foot: The right-left prevalence in spatial compatibility is still present. Perception \& Psychophysics, 38, 211-216.

Nicoletti, R., Umiltà, C., \& Ladavas, E. (1984). Compatibility due to the coding of the relative position of the effectors. Acta Psychologica, 57, 133-143.

Oldfield, S. R., \& Parker, S. P. A. (1984). Acuity of sound localisation: A topography of auditory space: I. Normal hearing conditions. Perception, 13, 581-600.

Posner, M. I., Nissen, M. J., \& KLeIN, R. (1976). Visual dominance: An information-processing account of its origins and significance. Psychological Review, 83, 157-171.

RICHARDS, J. T. (1978). Interitem structure and the facilitation of simultaneous comparison. Joumal of Experimental Psychology: Human Perception \& Performance, 4, 72-87.

ROYER, F. L. (1981). Detection of symmetry. Joumal of Experimental Psychology: Human Perception \& Performance, 7, 1186-1210.

SHoll, M. J., \& EGETH, H. E. (1981). Right-left confusion in the adult: A verbal labeling effect. Memory \& Cognition, 9, 339-350.

Simon, J. R. (1969). Reactions toward the source of stimulation. Journal of Experimental Psychology, 81, 174-176.

Simon, J. R., Mewaldt, S. P., Acosta, E., Jr., \& Hu, J. M. (1976). Processing auditory information: Interaction of two population stereotypes. Journal of Applied Psychology, 60, 354-358.

Simon, J. R., Sly, P. E., \& VilapakKam, S. (1981). Effect of compatibility of $S-R$ mapping on reactions toward the stimulus source. Acta Psychologica, 47, 63-81.

Teichner, W. H., \& KREBS, M. J. (1974). Laws of visual choice reaction time. Psychological Review, 81, 75-98.

UMILTÀ, C., \& NicoletTI, R. (1985). Attention and coding effects in S-R compatibility due to irrelevant spatial cues. In M. I. Posner \& O. S. M. Marin (Eds.), Attention and performance XI (pp. 457-471). Hillsdale, NJ: Erlbaum.

WALLACE, R. J. (1971). S-R compatibility and the idea of a response code. Journal of Experimental Psychology, 88, 354-360.

WALlace, R. J. (1972). Spatial S-R compatibility effects involving kinesthetic cues. Journal of Experimental Psychology, 93, 163-168.

(Manuscript received February 5, 1987; revision accepted for publication August 14, 1987.) 